\title{
An affine string vertex operator construction at an arbitrary level
}

\author{
R. W. Gebert \\ Institute for Advanced Study, School of Natural Sciences, Princeton, New Jersey 08540 \\ H. Nicolai \\ Max-Planck-Institut für Gravitationsphysik, Albert-Einstein-Institut, \\ Schlaatzweg 1, D-14473 Potsdam, Germany
}

(Received 13 January 1997; accepted for publication 9 April 1997)

\begin{abstract}
An affine vertex operator construction at an arbitrary level is presented which is based on a completely compactified chiral bosonic string whose momentum lattice is taken to be the (Minkowskian) affine weight lattice. This construction is manifestly physical in the sense of string theory, i.e., the vertex operators are functions of Del Giudice-Di Vecchia-Fubini (DFF) "oscillators" and the Lorentz generators, both of which commute with the Virasoro constraints. We therefore obtain explicit representations of affine highest weight modules in terms of physical (DDF) string states. This opens new perspectives on the representation theory of affine Kac-Moody algebras, especially in view of the simultaneous treatment of infinitely many affine highest weight representations of arbitrary level within a single state space as required for the study of hyperbolic Kac-Moody algebras. A novel interpretation of the affine Weyl group as the "dimensional null reduction", of the corresponding hyperbolic Weyl group is given, which follows upon reexpression of the affine Weyl translations as Lorentz boosts. (C) 1997 American Institute of Physics. [S0022-2488(97)03109-5]
\end{abstract}

\section{INTRODUCTION}

In this article we propose a generalization of the Frenkel-Kac-Segal (FKS) vertex operator realization of nontwisted affine Lie algebras at level one ${ }^{1,2}$ to an arbitrary level. This construction was originally based on the spatial compactification of a bosonic string whose momentum lattice is taken to be the (Euclidean) root lattice of a finite-dimensional simple Lie algebra of $A D E$ type. The Laurent coefficients (modes) of the tachyon vertex operators together with the string oscillators then constitute a basis of the affine algebra. This basis is not physical in the sense of string theory since, except for the zero mode, these operators do not commute with the Virasoro constraints. However, there is also a "covariant" version of the FKS construction," where the momentum lattice of the string is enlarged by a two-dimensional Minkowski lattice; then the zero mode operators are indeed physical in the sense of string theory and already by themselves form a basis of the affine algebra. Apparently it has not been generally appreciated so far that, apart from being manifestly physical, this construction is applicable to affine Lie algebras at arbitrary level and thus more general than the FKS construction. The characteristic feature of our model is that the momentum lattice of the string is taken to be the (Minkowskian) affine weight lattice. This model was recently exploited in Ref. 5 to construct an explicit representation of the affine Sugawara generators in terms of (transversal) Del Giudice-Di Vecchia-Fubini (DDF) operators at arbitrary level.

A main new result of this article is a string vertex operator realization of the affine CartanWeyl basis (in particular the step operators) at an arbitrary level in terms of physical (DDF) operators rather than ordinary string oscillators as in Refs. 3 and 4. Consequently, we can evaluate the action of these operators on any given physical state directly in terms of the DDF basis. This construction leads us to consider a new type of (level-dependent) physical field $\mathscr{B}^{\mu}(z)$, similar to the old Fubini-Veneziano field, $X^{\mu}(z)$, but where the ordinary string oscillators are replaced by level- $\ell$ transversal DDF oscillators. Apart from the center of mass coordinate, the fields $\mathscr{C}^{\mu}$ were 
already introduced in Ref. 5. Whereas the correct definition of the center of mass mode of $\mathscr{C}^{\mu} \mathrm{did}$ not matter in Ref. 5 , it is absolutely essential here; somewhat surprisingly, this definition turns out to involve the Lorentz generators $M_{\mu \nu}$, which are physical operators, rather than the operators $q^{\mu}$, which are not physical unlike the center of mass momenta $p^{\mu}$. The construction also requires a corresponding new type of "tachyon vertex operator" with $X^{\mu}$ replaced by $\mathscr{C}^{\mu}$; more general operators of this type presumably will be needed at a later stage.

The proper definition of the center of mass mode, and the corresponding replacement of a translation generator by a Lorentz transformation leads us to our second main result, namely a new interpretation of the affine Weyl group as a "dimensional null reduction" of the hyperbolic Weyl group. More specifically, this result hinges on re-expressing the so-called (affine) Weyl translations as Lorentz boosts. Consequently, these elements of the affine Weyl group should really be called "Weyl boosts." In this way it becomes obvious that the embeddings of the finite, affine and hyperbolic Weyl groups of the finite, affine and hyperbolic Kac-Moody algebras $\overline{\mathfrak{g}} \subset \mathfrak{g} \subset \hat{\mathfrak{g}}$, respectively (with the finite algebra $\overline{\mathfrak{g}}$ of rank $d-2$ ), are just the discrete analogs of the corresponding sequence of embeddings of the continuous groups $\mathrm{SO}(d-2) \subset \mathrm{ISO}(d-2) \subset \mathrm{SO}(d-1,1)$ into one another [see Eq. (4.16)]. Here $\operatorname{ISO}(d-2)$ is defined to be the subgroup of $\operatorname{SO}(d-1,1)$ leaving invariant a given lightlike vector, which in our case is just the affine null root $\delta$. We find it remarkable that this new description of the affine Weyl group is really forced upon us by the DDF approach, and this suggests that it is the truly natural interpretation of the known result that the affine Weyl group is a semidirect product of the finite Weyl group and the affine Weyl translations. While the affine Weyl transformations leave the level of a given representation fixed, one can in principle also consider level-changing boosts. As we expect such transformations to provide important new insights into the structure of hyperbolic Kac-Moody algebras and their Weyl groups we briefly discuss these generalizations in the last section.

We believe that the results presented in this article open new and promising perspectives for the theory of irreducible representations of affine Lie algebras, especially with regard to the problem of understanding hyperbolic Kac-Moody algebras (actually our main goal), where one must simultaneously deal with infinitely many inequivalent representations of arbitrary level. As shown in the present article, this aim can be achieved by embedding all the affine representation spaces into a single Fock space of physical states. Among the fascinating open problems for further study let us especially mention the idea of extending the present construction to "levelchanging vertex operators" (actually, this will be a generic feature when we go over to the hyperbolic extension of the affine Lie algebra), possibly also of more general-type than the tachyon-type vertex operators utilized here. Whereas the affine generators themselves involve only transversal DDF operators and thus contribute only transversal excitations to states within a single irreducible affine representation, the longitudinal DDF operators by construction map affine vacuum vectors into each other and will accordingly act as representation-changing (and, in general, even level-changing) operators. The latter have so far played no role in the representation theory of affine algebras, and are unnecessary as long as one deals only with one representation at a time. However, it is clear that a proper understanding of the longitudinal DDF operators is one of the keys to unraveling the mysteries of indefinite and hyperbolic Kac-Moody algebras.

\section{AFFINE WEIGHTS AND DDF OPERATORS}

We consider a nontwisted affine Lie algebra $\mathfrak{g}=\mathfrak{n}_{-} \oplus \mathfrak{h} \oplus \mathfrak{n}_{+}$(for general references on this subject see, e.g., Refs. 6 and 7) with underlying simple finite-dimensional Lie algebra $\overline{\mathfrak{g}}$ of type $A D E$ and with rank $d-2(d>2)$. The affine (respectively, finite) root lattice is denoted by $Q$ (respectively, $\bar{Q}$ ). The space of dominant integral affine weights is given by

$$
P_{+}:=\left\{\boldsymbol{\Lambda} \in \mathfrak{h}^{*} \mid \boldsymbol{\Lambda} \cdot \mathbf{r}_{I} \in \mathbb{Z}_{+}, 0 \leqslant I \leqslant d-2\right\}=\sum_{I=0}^{d-2} Z_{+} \boldsymbol{\Lambda}_{I}+\mathrm{C} \boldsymbol{\delta},
$$


where $\boldsymbol{\delta}$ is the affine null root, $\mathbf{r}_{I}$ are the affine simple roots, and $\boldsymbol{\Lambda}_{I}(0 \leqslant I \leqslant d-2)$ are the fundamental affine weights defined by $\boldsymbol{\Lambda}_{I} \cdot \mathbf{r}_{J}=\delta_{I J}$ and $\boldsymbol{\Lambda}_{I} \cdot \boldsymbol{\Lambda}_{0}=0$ for $0 \leqslant I, J \leqslant d-2$. For any dominant weight $\Lambda$ the level $\ell \in \mathbb{Z}$ is defined by (in comparison with Ref. 5 we have switched signs so as to be in unison with the standard conventions. ${ }^{6,7}$ )

$$
\ell:=\Lambda \cdot \boldsymbol{\delta} .
$$

By $L(\boldsymbol{\Lambda})$ we denote the corresponding integrable irreducible highest weight module over $\mathfrak{g}$. It is clear that $L(\boldsymbol{\Lambda}) \cong L(\boldsymbol{\Lambda}+z \boldsymbol{\delta})$ for all $z \in \mathrm{C}$. By putting

$$
\Lambda^{\prime}:=\Lambda+\frac{1}{\ell}\left(1-\frac{1}{2} \Lambda^{2}\right) \boldsymbol{\delta}
$$

for any $\boldsymbol{\Lambda} \in P_{+}$of nonzero level we thus obtain a "tachyonic" (i.e., $\boldsymbol{\Lambda}^{\prime 2}=2$ ) dominant integral affine weight which gives rise to a highest weight module $L\left(\boldsymbol{\Lambda}^{\prime}\right)$ isomorphic to $L(\boldsymbol{\Lambda})$. Without loss of generality we shall assume from now on that $\Lambda$ is some tachyonic dominant weight of positive level $\ell$.

Now let $\boldsymbol{\lambda}$ be any weight in $\Omega(\boldsymbol{\Lambda})$, the set of weights for $L(\boldsymbol{\Lambda})$. It ensues that (i) $\boldsymbol{\lambda}^{2} \in 2 Z$ and (ii) $\boldsymbol{\lambda}^{2} \leqslant \boldsymbol{\Lambda}^{2}=2$. To see this we note that $\boldsymbol{\lambda}=\boldsymbol{\Lambda}-\mathbf{r}$ for some $\mathbf{r} \in Q_{+}$. Then (i) follows from $\boldsymbol{\lambda}^{2}$ $=2(1-\boldsymbol{\Lambda} \cdot \mathbf{r})+\mathbf{r}^{2}$ by the fact that $\boldsymbol{\Lambda}$ is an integral weight and that $Q$ is an even lattice by assumption. To prove (ii), we use that $\boldsymbol{\lambda}$ is Weyl equivalent to a unique $\boldsymbol{\lambda}^{\prime} \in P_{+} \cap \Omega(\boldsymbol{\Lambda})$ with decomposition $\boldsymbol{\lambda}^{\prime}=\boldsymbol{\Lambda}-\mathbf{r}^{\prime}$ for some $\mathbf{r}^{\prime} \in Q_{+}$; hence $\boldsymbol{\lambda}^{2}=\boldsymbol{\lambda}^{\prime 2}=\boldsymbol{\Lambda}^{2}-\boldsymbol{\Lambda} \cdot \mathbf{r}^{\prime}-\boldsymbol{\lambda}^{\prime} \cdot \mathbf{r}^{\prime} \leqslant \boldsymbol{\Lambda}^{2}=2$ because both $\boldsymbol{\Lambda}$ and $\boldsymbol{\lambda}^{\prime}$ are dominant [cf. Ref. 6 (Proposition 11.4.a)].

These observations are crucial for the DDF construction to be described below: for any level$\ell$ weight $\boldsymbol{\lambda} \in \Omega(\boldsymbol{\Lambda})$ we define its DDF decomposition ${ }^{8}$ by

$$
\boldsymbol{\lambda}=\mathbf{a}-n \mathbf{k}_{\ell},
$$

where

$$
\mathbf{k}_{\ell}:=\frac{1}{\ell} \boldsymbol{\delta},
$$

and the vector $\mathbf{a}$ is uniquely determined by demanding $\mathbf{a}^{2}=2$. Thus $n=1-\frac{1}{2} \boldsymbol{\lambda}^{2}$, and by the above result, $n$ is always a non-negative integer as required by the DDF construction. We will refer to a as the "tachyonic level- $\ell$ vector" and to the corresponding state $|\mathbf{a}\rangle$ as the "tachyonic level- $\ell$ state" associated to $\boldsymbol{\lambda}$. Note that, for $\ell>1$, the tachyonic vector a occurring in Eq. (2.2) in general is not a weight for $L(\boldsymbol{\Lambda})$ because of the fractional coefficient in front of $\boldsymbol{\delta}$. Rather it will be used as an auxiliary vector in the construction below.

A central feature of our approach is the realization of the affine representation space $L(\Lambda)$ as a (tiny) subspace of a much bigger space $\mathscr{P}$ of physical string states, itself a subspace of a Fock space $\mathscr{F}$ which is the direct sum of irreducible Heisenberg modules created by the usual string oscillators from the ground states $|\boldsymbol{\lambda}\rangle \equiv \exp (i \boldsymbol{\lambda} \cdot \mathbf{q})|\mathbf{0}\rangle$ for arbitrary affine weights $\boldsymbol{\lambda}$. More precisely,

$$
\mathscr{F}:=\operatorname{span}\left\{\alpha_{-m_{1}}^{\mu_{1}} \cdots \alpha_{-m_{M}}^{\mu_{M}}|\boldsymbol{\lambda}\rangle \mid \boldsymbol{\lambda} \in \mathfrak{h}^{*}, m_{\mu}>0\right\},
$$

where the string oscillators $\alpha_{m}^{\mu}(m \in \mathbb{Z}, 0 \leqslant \mu \leqslant d-1)$ and the center of mass operators $q^{\mu}, p^{\mu}$ obey the standard commutation relations

$$
\left[\alpha_{m}^{\mu}, \alpha_{n}^{\nu}\right]=m \eta^{\mu \nu} \delta_{m+n, 0}, \quad\left[q^{\mu}, p^{\nu}\right]=i \eta^{\mu \nu}, \quad \alpha_{0}^{\mu} \equiv p^{\mu}
$$

with $p^{\mu}|\boldsymbol{\lambda}\rangle=\lambda^{\mu}|\boldsymbol{\lambda}\rangle$. To isolate the physical states, we introduce the Virasoro operators 


$$
L_{m}:=\frac{1}{2} \sum_{n \in \mathbb{Z}}: \boldsymbol{\alpha}_{n} \cdot \boldsymbol{\alpha}_{m-n}:
$$

which satisfy a Virasoro algebra with central charge $c=d$ [the normal-ordering $: \cdots:$ with respect to the string oscillators $\alpha_{m}^{\mu}$ in Eq. (2.5) is defined in the usual manner]. The space of physical string states $\mathscr{P} \subset \mathscr{F}$ is then defined as

$$
\mathscr{P}:=\left\{\psi \in \mathscr{F} \mid L_{0} \psi=\psi, L_{n} \psi=0 \forall n>0\right\} .
$$

As already indicated, we shall be interested in certain subspaces of $\mathscr{P}$; more specifically, the affine representation space $L(\boldsymbol{\Lambda})$ associated with the highest weight $\boldsymbol{\Lambda}$ will be embedded into the space

$$
\mathscr{P}(\boldsymbol{\Lambda})=\underset{\boldsymbol{\lambda} \in \Omega_{(\boldsymbol{\Lambda})}}{\bigoplus} \mathscr{P}^{(\boldsymbol{\lambda})} \subset \mathscr{P},
$$

where

$$
\mathscr{P}^{(\boldsymbol{\lambda})}:=\left\{\psi \in \mathscr{F} \mid L_{0} \psi=\psi, \quad L_{n} \psi=0 \forall n>0, p^{\mu} \psi=\lambda^{\mu} \psi, \quad 0 \leqslant \mu \leqslant d-1\right\}
$$

denotes the space of physical string states with momentum $\boldsymbol{\lambda}$.

An explicit realization of the physical states is afforded by the so-called DDF operators. ${ }^{9,10}$ To write them down we need the DDF decomposition (2.2) since these operators will always act on some tachyonic state $|\mathbf{a}\rangle$ associated with a given weight $\boldsymbol{\lambda}$ in the sense explained above. Furthermore, we need a set of polarization vectors $\boldsymbol{\xi}^{i} \equiv \boldsymbol{\xi}^{i}(\mathbf{a}) \equiv \boldsymbol{\xi}^{i}(\boldsymbol{\lambda})(1 \leqslant i \leqslant d-2)$ satisfying $\boldsymbol{\xi}^{i} \cdot \boldsymbol{\xi}^{j}$ $=\delta_{i j}$ and $\boldsymbol{\xi}^{i} \cdot \boldsymbol{\delta}=\boldsymbol{\xi}_{-}^{i} \cdot \mathbf{a}=0$, which constitute a basis for the complex vector space $\overline{\mathfrak{h}}^{*}$ dual to the Cartan subalgebra $\overline{\mathfrak{h}}$ of $\overline{\mathfrak{g}}$. The DDF operators are defined by ${ }^{9,10}$ [to make the notation less cumbersome, and contrary to the notational conventions of Ref. 5, we here suppress the label $\ell$ on the DDF operators in Eqs. (2.9) and (2.10) because this dependence is already implied by their dependence on a]

$$
\begin{gathered}
A_{m}^{i}(\mathbf{a}):=\oint \frac{d z}{2 \pi i} \boldsymbol{\xi}^{i}(\mathbf{a}) \cdot \mathbf{P}(z) \exp \left[i m \mathbf{k}_{\ell} \cdot \mathbf{X}(z)\right], \\
A_{m}^{-}(\mathbf{a}):=\oint \frac{d z}{2 \pi i}:\left[-\mathbf{a} \cdot \mathbf{P}(z)+\frac{m}{2} \frac{d}{d z} \ln \mathbf{k}_{\ell} \cdot \mathbf{P}(z)\right] \exp \left[i m \mathbf{k}_{\ell} \cdot \mathbf{X}(z)\right]: \\
-\frac{1}{2} \sum_{n \in Z} \underset{\times}{\times} A_{n}^{i}(\mathbf{a}) A_{m-n}^{i}(\mathbf{a})_{\times}^{\times}+2 \delta_{m 0} \mathbf{k}_{\ell} \cdot \mathbf{p}, \\
A_{m}^{+}(\mathbf{a}):=\oint \frac{d z}{2 \pi i} \mathbf{k}_{\ell} \cdot \mathbf{P}(z) \exp \left[i m \mathbf{k}_{\ell} \cdot \mathbf{X}(z)\right]=\delta_{m 0} \mathbf{k}_{\ell} \cdot \mathbf{p},
\end{gathered}
$$

for $m \in \mathbb{Z}, 1 \leqslant i \leqslant d-2$. Here we have used the well-known Fubini-Veneziano coordinate and momentum fields, respectively,

$$
\begin{gathered}
X^{\mu}(z):=q^{\mu}-i p^{\mu} \ln z+i \sum_{m \neq 0} \frac{1}{m} \alpha_{m}^{\mu} z^{-m}, \\
P^{\mu}(z):=i \frac{d}{d z} X^{\mu}(z)=\sum_{m \in \mathbb{Z}} \alpha_{m}^{\mu} z^{-m-1},
\end{gathered}
$$

and employed the standard normal-ordering $\underset{\times}{\times} \ldots \underset{\times}{\times}$ for the transversal DDF operators 


$$
{ }_{\times}^{\times} A_{m}^{i} A_{n \times}^{j \times}:=\left\{\begin{array}{ll}
A_{m}^{i} A_{n}^{j} & \text { for } m \leqslant n \\
A_{n}^{j} A_{m}^{i} & \text { for } m>n
\end{array} .\right.
$$

Let us recall from Ref. 5 that the shift of any polarization vector $\boldsymbol{\xi}^{i}(\mathbf{a}) \equiv \boldsymbol{\xi}^{i}(\boldsymbol{\lambda})$ along the $\boldsymbol{\delta}$ direction leaves the associated DDF operator $A_{m}^{i}(\mathbf{a})$ unchanged for $m \neq 0$, because the residue of a total derivative always vanishes. On the other hand, the difference $\boldsymbol{\xi}^{i}(\boldsymbol{\Lambda})-\boldsymbol{\xi}^{i}(\boldsymbol{\lambda})$ for any $\boldsymbol{\lambda}$ $\in \Omega(\boldsymbol{\Lambda})$ is, without loss of generality, always proportional to $\boldsymbol{\delta}$. Thus, for $m \neq 0$, we are effectively dealing with a single set of DDF operators $A_{m}^{i}(\boldsymbol{\Lambda})$ for the whole module $L(\boldsymbol{\Lambda})$; the zero mode operators do differ for different $\mathbf{a}$, however. For definiteness, we choose the polarization vectors to be $\boldsymbol{\xi}^{i}(\boldsymbol{\Lambda})$ throughout.

The above operators obey the commutation relations

$$
\begin{gathered}
{\left[A_{m}^{i}, A_{n}^{j}\right]=m \delta^{i j} \delta_{m+n, 0} \mathbf{k} / \mathbf{p},} \\
{\left[A_{m}^{-}, A_{n}^{i}\right]=0,} \\
{\left[A_{m}^{-}, A_{n}^{-}\right]=(m-n) A_{m+n}^{-}+\frac{26-d}{12} m\left(m^{2}-1\right) \delta_{m+n, 0} \mathbf{k}_{\ell} \cdot \mathbf{p} .}
\end{gathered}
$$

They are physical, i.e.,

$$
\left[L_{m}, A_{n}^{i}\right]=\left[L_{m}, A_{n}^{ \pm}\right]=0 . \quad \forall m, n \in \mathbb{Z}, 1 \leqslant i \leqslant d-2,
$$

and therefore map physical into physical states. Moreover, they constitute a spectrum-generating algebra for the string. In particular,

$$
\mathscr{P}^{(\boldsymbol{\lambda})}=\operatorname{span}\left\{A_{-m_{1}}^{i_{1}} \cdots A_{-m_{M}}^{i_{M}} A_{-n_{1}}^{-} \cdots A_{-n_{N}}^{-}|\mathbf{a}\rangle \mid \sum m_{\mu}+\sum n_{\nu}=1-\frac{1}{2} \boldsymbol{\lambda}^{2}\right\},
$$

for a DDF decomposition $\boldsymbol{\lambda}=\mathbf{a}-n \mathbf{k}_{\ell}$ of $\boldsymbol{\lambda}$ and for $i_{\mu}=1, \ldots, d-2, m_{\mu}>0, n_{1} \geqslant \cdots \geqslant n_{N} \geqslant 2$. Note that $A_{-1}^{-}|\mathbf{a}\rangle \propto L_{-1}\left|\mathbf{a}-\mathbf{k}_{\ell}\right\rangle$, i.e., $A_{-1}^{-}$generates null physical states which must be discarded.

\section{AFFINE VERTEX OPERATORS AT ARBITRARY LEVEL}

We introduce a linear map $\pi_{\boldsymbol{\Lambda}}: \mathfrak{g} \rightarrow \operatorname{End} \mathscr{P}(\boldsymbol{\Lambda})$ as follows:

$$
\begin{aligned}
& K \mapsto \boldsymbol{\delta} \cdot \mathbf{p}, \\
& d \mapsto \boldsymbol{\Lambda}_{0} \cdot \mathbf{p}, \\
& H_{m}^{i} \mapsto \oint \frac{d z}{2 \pi i} \boldsymbol{\xi}^{i}(\boldsymbol{\Lambda}) \cdot \mathbf{P}(z) \exp [\operatorname{im} \boldsymbol{\delta} \cdot \mathbf{X}(z)] \\
& E_{m}^{\mathbf{r}} \mapsto \oint \frac{d z}{2 \pi i}: \exp [i(\mathbf{r}+m \boldsymbol{\delta}) \cdot \mathbf{X}(z)]: c_{\mathbf{r}},
\end{aligned}
$$

with $\mathbf{r} \in \bar{\Delta}$ and thus $\mathbf{r}+m \boldsymbol{\delta} \in \Delta . c_{\mathbf{r}}$ denotes a cocycle factor satisfying $c_{\mathbf{r}} e^{i \mathbf{s} \cdot \mathbf{q}}=\boldsymbol{\epsilon}(\mathbf{r}, \mathbf{s}) e^{i \mathbf{s} \cdot \mathbf{q}} c_{\mathbf{r}}$ for some bimultiplicative two-cocycle $\epsilon$ normalized s.t. $\epsilon(\mathbf{0 , 0})=\epsilon(\mathbf{r},-\mathbf{r})=1$. Indeed, it is straightforward to check (see Refs. 3 and 4 ) that the above operators are physical, i.e.,

$$
\left[L_{m}, \pi_{\Lambda}(K)\right]=\left[L_{m}, \pi_{\Lambda}(d)\right]=\left[L_{m}, \pi_{\Lambda}\left(H_{n}^{i}\right)\right]=\left[L_{m}, \pi_{\Lambda}\left(E_{n}^{\mathbf{r}}\right)\right]=0
$$

for all $m, n \in \mathbb{Z}, \mathbf{r} \in \bar{\Delta}, 1 \leqslant i \leqslant d-2$. More precisely, for any $\boldsymbol{\lambda} \in \Omega(\boldsymbol{\Lambda})$ one has 


$$
\begin{gathered}
\pi_{\boldsymbol{\Lambda}}(K): \mathscr{P}^{(\boldsymbol{\lambda})} \rightarrow \mathscr{P}^{(\boldsymbol{\lambda})}, \\
\pi_{\boldsymbol{\Lambda}}(d): \mathscr{P}^{(\boldsymbol{\lambda})} \rightarrow \mathscr{P}^{(\boldsymbol{\lambda})}, \\
\pi_{\boldsymbol{\Lambda}}\left(H_{m}^{i}\right): \mathscr{P}^{(\boldsymbol{\lambda})} \rightarrow \mathscr{P}^{(\boldsymbol{\lambda}+m \boldsymbol{\delta})}, \\
\pi_{\boldsymbol{\Lambda}}\left(E_{m}^{\mathbf{r}}\right): \mathscr{P}^{(\boldsymbol{\lambda})} \rightarrow \mathscr{P}^{(\boldsymbol{\lambda}+\mathbf{r}+m \boldsymbol{\delta})} .
\end{gathered}
$$

Furthermore, the following relations hold:

$$
\begin{gathered}
{\left[\pi_{\boldsymbol{\Lambda}}\left(H_{m}^{i}\right), \pi_{\boldsymbol{\Lambda}}\left(H_{n}^{j}\right)\right]=\ell m \delta^{i j} \delta_{m+n, 0},} \\
{\left[\pi_{\boldsymbol{\Lambda}}\left(H_{m}^{i}\right), \pi_{\boldsymbol{\Lambda}}\left(E_{n}^{\mathbf{r}}\right)\right]=\left(\boldsymbol{\xi}^{i} \cdot \mathbf{r}\right) \pi_{\boldsymbol{\Lambda}}\left(E_{m+n}^{\mathbf{r}}\right),} \\
{\left[\pi_{\boldsymbol{\Lambda}}\left(E_{m}^{\mathbf{r}}\right), \pi_{\boldsymbol{\Lambda}}\left(E_{n}^{\mathbf{s}}\right)\right]=\left\{\begin{array}{l}
0 \text { if } \mathbf{r} \cdot \mathbf{s} \geqslant 0 \\
\boldsymbol{\epsilon}(\mathbf{r}, \mathbf{s}) \pi_{\boldsymbol{\Lambda}}\left(E_{m+n}^{\mathbf{r}+\mathbf{s}}\right) \text { if } \mathbf{r} \cdot \mathbf{s}=-1, \\
\pi_{\boldsymbol{\Lambda}}\left(H_{m+n}^{\mathbf{r}}\right)+\ell m \delta_{m+n, 0} \quad \text { if } \mathbf{r} \cdot \mathbf{s}=-2
\end{array}\right.} \\
{\left[\pi_{\boldsymbol{\Lambda}}(K), \pi_{\boldsymbol{\Lambda}}(x)\right]=0 \quad \forall x \in \mathfrak{g},} \\
{\left[\pi_{\boldsymbol{\Lambda}}(d), \pi_{\boldsymbol{\Lambda}}\left(H_{m}^{i}\right)\right]=m \pi_{\Lambda}\left(H_{m}^{i}\right),} \\
{\left[\pi_{\boldsymbol{\Lambda}}(d), \pi_{\boldsymbol{\Lambda}}\left(E_{m}^{\mathbf{r}}\right)\right]=m \pi_{\boldsymbol{\Lambda}}\left(E_{m}^{\mathbf{r}}\right) .}
\end{gathered}
$$

Hence $\pi_{\Lambda}$ defines a level- $\ell$ vertex operator realization of $\mathfrak{g}$ on $\mathscr{P}(\boldsymbol{\Lambda})$. By identifying the vacuum vector $v_{\boldsymbol{\Lambda}}$ in $L(\boldsymbol{\Lambda})$ with the tachyonic ground state $|\boldsymbol{\Lambda}\rangle$ in $\mathscr{P}(\boldsymbol{\Lambda})$, we conclude that

$$
L(\mathbf{\Lambda}) \hookrightarrow \mathscr{P}(\boldsymbol{\Lambda}) .
$$

To see this, we first write down the realization of the Chevalley-Serre generators, viz.

$$
\begin{gathered}
e_{i}:=\pi_{\boldsymbol{\Lambda}}\left(E_{0}^{\mathbf{r}_{i}}\right), \quad f_{i}:=-\pi_{\boldsymbol{\Lambda}}\left(E_{0}^{-\mathbf{r}_{i}}\right), \quad h_{i}:=\pi_{\boldsymbol{\Lambda}}\left(\mathbf{r}_{i} \cdot \mathbf{H}_{0}\right)=\mathbf{r}_{i} \cdot \mathbf{p}, \quad \text { for } 1 \leqslant i \leqslant d-2, \\
e_{0}:=\pi_{\Lambda}\left(E_{1}^{-\boldsymbol{\theta}}\right), \quad f_{0}:=-\pi_{\boldsymbol{\Lambda}}\left(E_{-1}^{\boldsymbol{\theta}}\right), \quad h_{0}:=\pi_{\boldsymbol{\Lambda}}\left(K-\boldsymbol{\theta} \cdot \mathbf{H}_{0}\right)=\mathbf{r}_{0} \cdot \mathbf{p},
\end{gathered}
$$

where $\boldsymbol{\theta}$ denotes the highest root in $\bar{\Delta}$. Ten we have to verify [see, e.g., Ref. 6 (Corollary 10.4)] both the vacuum vector conditions

$$
e_{I}|\boldsymbol{\Lambda}\rangle=0 \quad \text { for } 0 \leqslant I \leqslant d-2,
$$

and the null vector conditions

$$
f_{I}^{1+\mathbf{r}_{I} \cdot \Lambda}|\Lambda\rangle=0 \quad \text { for } 0 \leqslant I \leqslant d-2
$$

From Eq. (3.2) we infer that

$$
e_{I}: \mathscr{P}^{(\boldsymbol{\lambda})} \rightarrow \mathscr{P}^{\left(\boldsymbol{\lambda}+\mathbf{r}_{I}\right)}, \quad f_{I}: \mathscr{P}^{(\boldsymbol{\lambda})} \rightarrow \mathscr{P}^{\left(\boldsymbol{\lambda}-\mathbf{r}_{I}\right)} \quad \text { for } 0 \leqslant I \leqslant d-2 .
$$

Hence $e_{I}|\boldsymbol{\Lambda}\rangle$ has at least eigenvalue 2 for $L_{0}$ because $\Lambda^{2}=\mathbf{r}_{I}^{2}=2$ and $\boldsymbol{\Lambda}$ is dominant, so that ( $\boldsymbol{\Lambda}$ $+\mathbf{r})^{2} \geqslant 4$; but this contradicts the fact that $e_{I}|\boldsymbol{\Lambda}\rangle$ is a physical state [cf. Eq. (2.8)], hence $e_{I}|\mathbf{\Lambda}\rangle$ must be zero. The null vectors $f_{I}^{1+\mathbf{r}_{I} \cdot \boldsymbol{\Lambda}}|\boldsymbol{\Lambda}\rangle$ vanish by the same argument since $\frac{1}{2}\left[\boldsymbol{\Lambda}-\left(1+\mathbf{r}_{I}\right.\right.$ 
$\left.\cdot \boldsymbol{\Lambda}) \mathbf{r}_{I}\right]^{2}=2+\boldsymbol{\Lambda} \cdot \mathbf{r}_{I}$. In other words, the null vectors are really zero in our approach. The space $\mathscr{P}^{(\boldsymbol{\lambda})}$ must not be confused with the weight space $L(\boldsymbol{\Lambda})_{\boldsymbol{\lambda}}$, the space of states with weight $\boldsymbol{\lambda}$ in the representation; rather, we have the (in general proper) inclusion

$$
L(\boldsymbol{\Lambda})_{\boldsymbol{\lambda}} \hookrightarrow \mathscr{P}^{(\boldsymbol{\lambda})} .
$$

If we make use of the observation that in Eq. (3.1) only transversal linear combinations of the string oscillators and consequently transversal DDF operators can occur, we conclude that

$$
\operatorname{mult}_{\boldsymbol{\Lambda}}(\boldsymbol{\lambda})=\operatorname{dim} L(\boldsymbol{\Lambda})_{\boldsymbol{\lambda}} \leqslant \operatorname{dim} \mathscr{P}_{\text {transv. }}^{(\boldsymbol{\lambda})}=p_{d-2}\left(1-\frac{1}{2} \boldsymbol{\lambda}^{2}\right) .
$$

This is a universal estimate for the weight multiplicities of any irreducible affine highest weight module which seems to be new. For $\ell=1$ this bound is known to be saturated; ${ }^{1}$ at a higher level, however, the formula may constitute only a crude upper bound. In general, there are "missing states," namely the physical states which lie in $\mathscr{P}^{(\boldsymbol{\lambda})}$ but not in $L(\boldsymbol{\Lambda})_{\boldsymbol{\lambda}}$. Note, however, that these have nothing to do with the above null vectors.

We also note that

$$
\pi_{\Lambda}\left(H_{m}^{i}\right)=A_{/ m}^{i}(\Lambda),
$$

which shows that the transversal DDF operators $A_{/ m}^{i}(\boldsymbol{\Lambda})$ occur not only as part of the spectrum generating algebra for the physical string states but also as homogeneous Heisenberg subalgebra of the affine algebra. One might therefore ask whether it is possible to rewrite the step operators $\pi_{\Lambda}\left(E_{m}^{\mathbf{r}}\right)$ also in a manifestly physical form in terms of these DDF operators. Indeed, this is possible if in addition one uses the Lorentz generators. To this end we introduce the transversal coordinate field

$$
\mathscr{C}_{\Lambda}^{i}(z) \equiv \mathscr{C}^{i}(z):=\left(\boldsymbol{\xi}^{i}\right)_{\mu}\left(\mathbf{k}_{\ell}\right)_{\nu} M^{\mu \nu}-i\left(\boldsymbol{\xi}^{i} \cdot \mathbf{p}\right) \ln z+i \sum_{m \neq 0} \frac{1}{m} A_{m}^{i}(\boldsymbol{\Lambda}) z^{-m},
$$

where

$$
M^{\mu \nu}:=q^{\mu} p^{\nu}-q^{\nu} p^{\mu}-i \sum_{n \neq 0} \frac{1}{n} \alpha_{-n}^{[\mu} \alpha_{n}^{\nu]}
$$

are the Lorentz generators, and the transversal momentum field

$$
\mathscr{P}_{\Lambda}^{i}(z) \equiv \mathscr{P}^{i}(z):=i \frac{d}{d z} \mathscr{O}^{i}(z)=\sum_{m \in Z} A_{m}^{i}(\boldsymbol{\Lambda}) z^{-m-1}
$$

(where we again do not indicate the dependence on the level explicitly.) Note that the center of mass coordinate in Eq. (3.15) is $\left(\boldsymbol{\xi}^{i}\right)_{\mu}\left(\mathbf{k}_{\ell}\right)_{\nu} M^{\mu \nu}$ rather than $q^{i}$ as one might have naively guessed. This choice is forced upon us by the requirement that the field $\mathscr{X}^{i}(z)$ should be physical: since

$$
\left[L_{m}, M^{\mu \nu}\right]=0,
$$

we have, with definition (3.15),

$$
\left[L_{m}, \mathscr{X}^{i}(z)\right]=0,
$$

whereas Eq. (3.19) would not vanish if the zero mode were $q^{i}$. Second, substituting $\left(\boldsymbol{\xi}^{i}\right)_{\mu}\left(\mathbf{k}_{\ell}\right)_{\nu} M^{\mu \nu}$ for $q^{i}$ amounts to a replacement of a translation generator (in momentum space) by a Lorentz rotation. As we will see, this is precisely what is required because our new expres- 
sions are defined in terms of DDF operators which shift the momentum by a vector proportional to $\mathbf{k}_{/}$whereas Eq. (3.1) is defined in terms of ordinary string oscillators $\alpha_{m}^{\mu}$ which do not shift momentum. There is a corresponding reinterpretation of the affine Weyl translation by a Lorentz boost (see Sec. IV).

Observe also that we have defined the new field $\mathscr{Q}^{i}(z)$ so far only for transversal indices. However, our definition can be generalized to the longitudinal components $\mathscr{X}^{ \pm}$by means of the operators $A_{m}^{ \pm}$defined in Eqs. (2.10) and (2.11), respectively. Note that $A_{m}^{+} \equiv 0$ for all $m \neq 0$, just as in light cone gauge string theory. Although we will make no use of the components $\mathscr{X}^{ \pm}$in this article, we expect them to become relevant in future generalizations involving level-changing operators (see Sec. V). We note that fields (3.15) are transcendental expressions in terms of the standard oscillator basis.

Next we establish the relation between the "old" step operators $\pi_{\Lambda}\left(E_{m}^{\mathbf{r}}\right)$ defined in Eq. (3.1) and a set of new ones which manifestly depend on the DDF operators. The "new" level- $\ell$ step operators are defined by

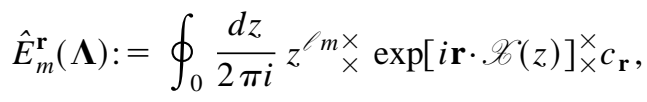

where we use the standard normal ordering (2.14) for the Heisenberg oscillators and where the cocycle factors $c_{\mathbf{r}}$, which are functions of momentum, were explained after Eq. (3.1) and are the same as in Ref. 1. The operators (3.20) will permit us to evaluate the action of the step operators directly in terms of the DDF basis.

Theorem 1: On the representation space $L(\Lambda)$, we have

$$
\pi_{\Lambda}\left(E_{m}^{\mathbf{r}}\right)=\hat{E}_{m}^{\mathbf{r}}(\boldsymbol{\Lambda})
$$

where the operators $\pi_{\Lambda}\left(E_{m}^{\mathbf{r}}\right)$ and $\hat{E}_{m}^{\mathbf{r}}(\boldsymbol{\Lambda})$ are defined, respectively, in Eqs. (3.1) and (3.20). Consequently, the operators $\hat{E}_{m}^{\mathrm{r}}(\boldsymbol{\Lambda}), A_{\ell m}^{i}(\boldsymbol{\Lambda}), \boldsymbol{\delta} \cdot \mathbf{p}$, and $\boldsymbol{\Lambda}_{0} \cdot \mathbf{p}$ realize the affine algebra at level $\ell$ on $\mathscr{P}(\boldsymbol{\Lambda})$ in terms of the transversal Heisenberg algebra spanned by the $A_{m}^{i}$ 's.

Proof: By construction, the operators $\hat{E}_{m}^{\mathbf{r}}$ are physical. The DDF operator $A_{n}^{i}$ shifts the momentum by $n \mathbf{k}_{\ell}$, and since the residue in Eq. (3.20) picks up $1+\ell m+\mathbf{r} \cdot \mathbf{a}$ of such modes for $\hat{E}_{m}^{\mathbf{r}}$ (a denotes the eigenvalue of $\mathbf{p}$ ), the contribution of the DDF oscillators to the shift of momentum will be $(1+\ell m+\mathbf{r} \cdot \mathbf{a}) \mathbf{k}_{\ell}$. On the other hand, the zero mode involving the Lorentz generators provides a momentum shift by $\mathbf{r}-(1+\mathbf{r} \cdot \mathbf{a}) \mathbf{k}_{\ell} \equiv{ }^{\left[{ }^{[}\right]} t_{\mathbf{r}}(\mathbf{a})-\mathbf{a}$, so that in total $\hat{E}_{m}^{\mathbf{r}}$ maps $\mathscr{P}^{(\boldsymbol{\lambda})}$ into $\mathscr{P}^{(\boldsymbol{\lambda}+\mathbf{r}+m \boldsymbol{\delta})}$ as required. The momentum shift ${ }^{[\ell]} t_{\mathbf{r}}(\mathbf{a})$ is just a Weyl translation, and we will return to this point in Sec. IV [see Eq. (4.4)].

Next, we have to check that the new step operators satisfy the required commutation relations, and this part of the proof is very similar to the corresponding one for the FKS construction. From the last observation we immediately get

$$
\left[\mathbf{v} \cdot \mathbf{p}, \hat{E}_{m}^{\mathbf{r}}\right]=\mathbf{v} \cdot(\mathbf{r}+m \boldsymbol{\delta}) \hat{E}_{m}^{\mathbf{r}},
$$

for any $\mathbf{v} \in \mathfrak{h}^{*}$, which yields the correct commutation relations with $\pi_{\Lambda}(K), \pi_{\Lambda}(d)$, and $\pi_{\Lambda}\left(H_{0}^{i}\right)$. By the use of Eqs. (3.14), (4.14), and (2.15) we obtain, for $m \neq 0$,

$$
\left[\pi_{\Lambda}\left(H_{m}^{i}\right), i \mathscr{X}_{\Lambda}^{j}(z)\right]=\delta^{i j} z^{m}
$$

from which Eq. (3.4) follows. Now, let us work out the commutator of two step operators which amounts to calculating the operator product of normal-ordered exponentials of the transversal coordinate field, namely, 


$$
\left[\hat{E}_{m}^{\mathbf{r}}, \hat{E}_{n}^{\mathbf{s}}\right]=\boldsymbol{\epsilon}(\mathbf{r}, \mathbf{s}) \oint_{0} \frac{d w}{2 \pi i} \oint_{w} \frac{d z}{2 \pi i} z^{\ell m} w_{\times}^{\ell n \times} \exp [i \mathbf{r} \cdot \mathscr{B}(z)]_{\times \times}^{\times \times} \exp [i \mathbf{s} \cdot \mathscr{B}(w)]_{\times}^{\times} c_{\mathbf{r}+\mathbf{s}} .
$$

We split the transversal coordinate field as follows:

$$
\mathscr{C}_{\Lambda}^{i}(z)=\mathscr{C}^{i}<(z)+Q^{i}-i P^{i} \ln z+\mathscr{C}_{>}^{i}(z)
$$

where

$$
\mathscr{C}_{\lessgtr}^{i}(z):=i \sum_{m \lessgtr 0} \frac{1}{m} A_{m}^{i}(\boldsymbol{\Lambda}) z^{-m}, \quad Q^{i}:=\left(\boldsymbol{\xi}^{i}\right)_{\mu}(\mathbf{k} /)_{\nu} M^{\mu \nu}, \quad P^{i}:=\boldsymbol{\xi}^{i} \cdot \mathbf{p}
$$

so that we can write the step operators explicitly as

$$
\hat{E}_{m}^{\mathbf{r}}=\oint \frac{d z}{2 \pi i} \exp \left[i \mathbf{r} \cdot \mathscr{C}_{<}(z)\right] e^{i \mathbf{r} \cdot \mathbf{Q}_{z} \mathbf{r} \cdot \mathbf{P}} \exp \left[i \mathbf{r} \cdot \mathscr{C}_{>}(z)\right] c_{\mathbf{r}}
$$

Using Eq. (2.15) and the relation $\left[Q^{i}, P^{j}\right]=i \delta^{i j}$, which is valid on the level- $\ell$ subspace $\mathscr{P}(\boldsymbol{\Lambda})$ only, we find that

$$
\begin{gathered}
\exp \left[i \mathbf{r} \cdot \mathscr{C}_{>}(z)\right] \exp \left[i \mathbf{s} \cdot \mathscr{C}_{<}(w)\right]=\left(1-\frac{w}{z}\right)^{\mathbf{r} \cdot \mathbf{s}} \exp \left[i \mathbf{s} \cdot \mathscr{C}_{<}(w)\right] \exp \left[i \mathbf{r} \cdot \mathscr{C}_{>}(z)\right] \quad(|z|>|w|), \\
z^{\mathbf{r} \cdot \mathbf{P}} e^{i \mathbf{s} \cdot \mathbf{Q}}=z^{\mathbf{r} \cdot \mathbf{s}} e^{i \mathbf{s} \cdot \mathbf{Q}} z^{\mathbf{r} \cdot \mathbf{P}}, \\
e^{i \mathbf{r} \cdot \mathbf{Q}} c_{\mathbf{r}} e^{i \mathbf{s} \cdot \mathbf{Q}} c_{\mathbf{s}}=\boldsymbol{\epsilon}(\mathbf{r}, \mathbf{s}) e^{i(\mathbf{r}+\mathbf{s}) \cdot \mathbf{Q}_{c}} c_{\mathbf{r}+\mathbf{s}}
\end{gathered}
$$

Thus, we have

$$
\begin{aligned}
{\left[\hat{E}_{m}^{\mathbf{r}}, \hat{E}_{n}^{\mathbf{s}}\right]=} & \boldsymbol{\epsilon}(\mathbf{r}, \mathbf{s}) \oint_{0} \frac{d w}{2 \pi i} \oint_{w} \frac{d z}{2 \pi i}\left\{z^{\ell m} w^{\ell n}(z-w)^{\mathbf{r} \cdot \mathbf{s}} \exp \left[i \mathbf{r} \cdot \mathscr{C}_{<}(z)+i \mathbf{s} \cdot \mathscr{C}_{<}(w)\right]\right. \\
& \left.\times e^{i(\mathbf{r}+\mathbf{s}) \cdot \mathbf{Q}_{z} \mathbf{r} \cdot \mathbf{P}} w^{\mathbf{s} \cdot \mathbf{P}} \exp \left[i \mathbf{r} \cdot \mathscr{C}_{>}(z)+i \mathbf{s} \cdot \mathscr{C}_{>}(w)\right]\right\} c_{\mathbf{r}+\mathbf{s}} .
\end{aligned}
$$

It is clear that the commutator vanishes for $\mathbf{r} \cdot \mathbf{s} \geqslant 0$. For $\mathbf{r} \cdot \mathbf{s}=-1$, we have $\mathbf{r}+\mathbf{s} \in \bar{\Delta}$. Furthermore, the contour integral of $z$ around $w$ then has the effect of setting $z=w$ in the integrand due to the simple pole, and the result $\epsilon(\mathbf{r}, \mathbf{s}) \hat{E}_{m+n}^{\mathbf{r}+\mathbf{s}}$ follows. The case $\mathbf{r} \cdot \mathbf{s}=-2$ is equivalent to $\mathbf{s}=-\mathbf{r}$ and corresponds to a second order pole at $z=w$ of the integrand. Cauchy's theorem then yields the required result, viz.

$$
\begin{aligned}
{\left[\hat{E}_{m}^{\mathbf{r}}, \hat{E}_{n}^{\mathbf{s}}\right] } & =\oint_{0} \frac{d w}{2 \pi i} w^{\ell n} \frac{d}{d z}\left[z^{\ell m}+i \mathbf{r} \cdot \mathscr{C}_{<}(z)+\left(\frac{z}{w}\right)^{\mathbf{r} \cdot \mathbf{P}}+i \mathbf{r} \cdot \mathscr{C}_{>}(z)\right]_{z=w} \\
& =\oint_{0} \frac{d w}{2 \pi i} w^{\ell(m+n)}\left[\mathbf{r} \cdot \mathscr{P}(w)+\ell m w^{-1}\right]=A_{m+n}^{\mathbf{r}}+\ell m \delta_{m+n, 0}
\end{aligned}
$$

Finally, we have to verify that $\hat{E}_{m}^{\mathbf{r}}$ really gives the same result as $\pi_{\Lambda}\left(E_{m}^{\mathbf{r}}\right)$ in terms of the string oscillators. For this purpose, we re-express the DDF operators for a given transversal physical state in terms of ordinary string oscillators. Then the leading oscillator contributions are the same because any product of DDF operators $A_{m}^{i}$ differs from the corresponding product of string oscillators $\alpha_{m}^{i}$ only by terms all of which involve at least one lightlike oscillator $\boldsymbol{\delta} \cdot \boldsymbol{\alpha}_{-n}$ with the null root $\boldsymbol{\delta}$ [this statement is no longer true for longitudinal DDF operators as can be seen by 
simple inspection of Eqs. (A7)-(A10) given in Ref. 8]. Moreover, the Lorentz generators do not contribute to the leading oscillator terms. Since any physical state is uniquely determined by its leading oscillators, the result follows.

We emphasize again that the equality stated in the theorem holds only on the subspace $\mathscr{P}(\boldsymbol{\Lambda}) \subset \mathscr{P}$, but not on the whole physical state space $\mathscr{P}$. This is because we must utilize the relation $\mathbf{k}_{\ell} \cdot \mathbf{a}=\mathbf{k}_{\ell} \cdot \boldsymbol{\lambda}=1$ for all $\boldsymbol{\lambda} \in \Omega(\boldsymbol{\Lambda})$ in the proof. We note that an analogous result would hold for the original FKS construction if one makes the simple replacement $z^{m} \rightarrow z^{\ell m}$ (see Ref. 11 where this observation was made in the context of parafermions). However, in our approach, the level- $\ell$ representation space is a subspace of a much bigger space, the space of all physical states of arbitrary level, which enables us to treat infinitely many irreducible representations simultaneously (as required by a representation theoretic approach to hyperbolic Kac-Moody algebras).

One of the nice features of the above realization is that it allows us to simply understand what is special about the basic representation, i.e., level $\ell=1$ : only in this case is it possible to express the step operators entirely in terms of the homogeneous Heisenberg subalgebra spanned by the $\pi_{\Lambda}\left(H_{m}^{i}\right)$ 's, for $|\ell|>1$, the step operators cannot be expressed in terms of the operators $A_{\ell m}^{i}$ alone. As a result we again have missing states, namely physical states which cannot be "reached" by applying step operators successively to the tachyonic vacuum vector $v_{\boldsymbol{\Lambda}} \equiv|\boldsymbol{\Lambda}\rangle$ that defines the representation. As we pointed out already, these missing states must not be confused with the null vectors of the conventional approach. The consequences of our new formulation for the computation of affine characters is an intriguing problem for further study.

As an application of Theorem 1 we can immediately rederive the new expression for the affine Sugawara generators given in Ref. 5. Recall that in terms of the affine Cartan-Weyl basis (3.1) these are given by

$$
\mathscr{B}_{m}^{[\ell]}:=\frac{1}{2\left(\ell+h^{\vee}\right)} \sum_{n \in \mathbb{Z}}\left[\sum_{i=1}^{d-2}{ }_{\circ}^{\circ} \pi_{\Lambda}\left(H_{n}^{i}\right) \pi_{\Lambda}\left(H_{m-n}^{i}\right)_{\circ}^{\circ}+\sum_{\mathbf{r} \in \bar{\Delta}}{ }_{\circ}^{\circ} \pi_{\Lambda}\left(E_{n}^{\mathbf{r}}\right) \pi_{\Lambda}\left(E_{m-n}^{-\mathbf{r}}\right)_{\circ}^{\circ}\right],
$$

where $h^{\vee}$ denotes the dual Coxeter number of $\overline{\mathfrak{g}}$. The new normal-ordering symbol ${ }_{\circ}^{\circ}{ }_{\circ}^{\circ}$ refers to the mode indices of the affine generators; for the operators $\pi_{\Lambda}\left(H_{n}^{i}\right)$ (but not for the step operators!) it is the same as Eq. (2.14) by Eq. (3.14). The operators $\mathscr{L}_{m}^{\left[{ }^{\prime}\right]}$ are well known to generate a Virasoro algebra (see, e.g., Ref. 12, and references therein)

$$
\left[\mathscr{O}_{m}^{[\ell}, \mathscr{O}_{n}^{[\bigcap]}\right]=(m-n) \mathscr{L}_{m+n}^{[\bigcap]}+\frac{c(\ell)}{12}\left(m^{3}-m\right) \delta_{m+n, 0} \pi_{\Lambda}(K),
$$

with central charge

$$
c(\ell):=\frac{\ell \operatorname{dim} \mathfrak{g}}{\ell+h^{\vee}} .
$$

They act as outer derivations on the affine Lie algebra according to

$$
\left[\mathscr{L}_{m}^{[\ell]}, A_{\ell n}^{i}\right]=-n A_{\ell(m+n)}^{i}, \quad\left[\mathscr{L}_{m}^{[\ell}, \hat{E}_{n}^{\mathbf{r}}\right]=-n \hat{E}_{m+n}^{\mathbf{r}} .
$$

In particular, we observe that $\mathscr{L}_{0}^{[\ell]}=-\pi_{\Lambda}(d)$. By construction, the Sugawara generators are physical, viz.

$$
\left[\mathscr{L}_{m}^{[\ell}, L_{n}\right]=0 \quad \forall m, n \in \mathbb{Z} .
$$

Now let $\zeta:=e^{2 \pi i l \ell}$ (or any other primitive $\ell$ th root of unity). We have ${ }^{5}$ the following corollary.

Corollary 1: The operators $\mathscr{L}_{m}^{[\ell]}$ can be directly expressed in terms of the transversal Heisenberg algebra by 


$$
\begin{aligned}
& \mathscr{L}_{m}^{[\ell]}=\frac{1}{2 \ell} \sum_{n \in Z} \sum_{i=1}^{d-2}{ }_{\times}^{\times} A_{\ell n}^{i} A_{\ell(m-n) \times}^{i}+\frac{h^{\vee}}{2 \ell\left(\ell+h^{\vee}\right)} \sum_{n \neq 0(\ell)} \sum_{i=1}^{d-2}{ }_{\times}^{\times} A_{n}^{i} A_{\ell m-n \times}^{i} \times \\
& +\frac{\left(\ell^{2}-1\right)(d-2) h^{\vee}}{24 \ell\left(\ell+h^{\vee}\right)} \delta_{m, 0}-\frac{1}{2 \ell\left(\ell+h^{\vee}\right)} \sum_{\mathbf{r} \in \bar{\Delta}} \sum_{p=1}^{\ell-1} \frac{1}{\left|\zeta^{p}-1\right|^{2}} \\
& \times \oint_{0} \frac{d z}{2 \pi i} z^{\ell m-1 \times} \exp \left\{i \mathbf{r} \cdot\left[\mathscr{C}\left(\zeta^{p} z\right)-\mathscr{C}(z)\right]\right\}_{\times}^{\times} \text {. }
\end{aligned}
$$

Proof: Using the operator expansion in the proof of Theorem 1 we get

$$
\begin{aligned}
& \sum_{n \in Z}{ }_{0}^{0} \hat{E}_{-n}^{\mathbf{r}} \hat{E}_{m+n^{\circ}}^{-\mathbf{r}} \equiv \sum_{n \geqslant 0} \hat{E}_{-n}^{\mathbf{r}} \hat{E}_{m+n}^{-\mathbf{r}}+\sum_{n>0} \hat{E}_{m-n}^{\mathbf{r}} \hat{E}_{n}^{-\mathbf{r}} \\
& =\left\{\oint \frac{d z}{2 \pi i} \oint_{|z|>|w|} \frac{d w}{2 \pi i}-\oint \frac{d z}{2 \pi i} \oint_{|z|<|w|} \frac{d w}{2 \pi i}\right\}(z-w)^{-2} \sum_{n \geqslant 0} z^{-\ell n} w^{\ell(m+n)} \\
& \times\left(\frac{z}{w}\right)^{\mathbf{r} \cdot \mathbf{P}} \exp \left\{i \mathbf{r} \cdot\left[\mathscr{C}_{<}(z)-\mathscr{C}_{<}(w)\right]\right\} \exp \left\{i \mathbf{r} \cdot\left[\mathscr{C}_{>}(z)-\mathscr{C}_{>}(w)\right]\right\} \\
& =\oint_{0} \frac{d w}{2 \pi i} \sum_{p=1}^{\ell} \oint_{w_{p}} \frac{d z}{2 \pi i}\left\{\frac{z^{\ell} w^{\ell m}}{(z-w)^{2}\left(z^{\ell}-w^{\ell}\right)} \stackrel{\times}{\times} \exp \{i \mathbf{r} \cdot[\mathscr{C}(z)-\mathscr{C}(w)]\}_{\times}^{\times}\right\},
\end{aligned}
$$

where $w_{p}:=\zeta^{p} w$. With the identity

$$
\frac{z^{\ell}-w^{\ell}}{z-w_{p}}=z^{\ell-1}+z^{\ell-2} w_{p}+\cdots+z w_{p}^{\ell-2}+w_{p}^{\ell-1}=: F\left(z, w_{p}\right),
$$

the sum over the poles at $z=w_{p}$ for $1 \leqslant p \leqslant \ell-1$ immediately yields the third term in Eq. (3.27). As regards the pole at $z=w$, we have to evaluate

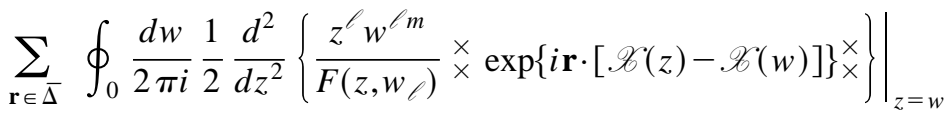

$$
\begin{aligned}
& =\frac{1}{2} \sum_{\mathbf{r} \in \bar{\Delta}} \oint_{0} \frac{d w}{2 \pi i}\left\{w^{\ell m} \frac{d^{2}}{d z^{2}}\left[\frac{z^{\ell}}{F\left(z, w_{\ell}\right)}\right]_{z=w}+\frac{1}{\ell} w^{\ell m+1 \times} \times\left[\frac{d}{d w} i \mathbf{r} \mathscr{C}(w)\right]_{\times}^{2 \times}\right\},
\end{aligned}
$$

where the terms linear in $\mathbf{r}$ drop out due to the sum over both positive and negative roots. Using Eq. (3.17) and the fact that

$$
\sum_{\mathbf{r} \in \bar{\Delta}} \mathbf{r} \otimes \mathbf{r}=2 h^{\vee} \sum_{i=1}^{d-2} \boldsymbol{\xi}^{i} \otimes \boldsymbol{\xi}^{i}
$$

the second term is seen to give a contribution

$$
\frac{h^{\vee}}{2 \ell\left(\ell+\ell^{\vee}\right)} \sum_{n \in Z} \sum_{i=1}^{d-2}{ }_{\times}^{\times} A_{n}^{i} A_{\ell m-n \times}^{i} \underset{\times}{\times}
$$

in the formula for the Sugawara operators. Finally, a straightforward calculation yields 


$$
\frac{1}{2} \frac{d^{2}}{d z^{2}}\left[\frac{z^{\ell}}{F\left(z, w_{\ell}\right)}\right]_{z=w}=\frac{\ell^{2}-1}{12 \ell w}
$$

which, together with $|\bar{\Delta}|=(d-2) h^{\vee}$, leads to the constant term in Eq. (3.27).

\section{THE AFFINE WEYL GROUP}

We now return to the remarks made at the beginning of the proof of Theorem 1. As noted there, the momentum shift effected by the step operator (3.20) on a given state is the combination of the shifts effected by the DDF operators (which are always along the null root $\boldsymbol{\delta}$ ) and a zero mode contribution, such that the total shift coincides with the one obtained for the original step operator of Eq. (3.2). Furthermore, we observed that the so-called (affine) Weyl translations naturally appeared there; the latter are designated by $t_{\mathbf{r}} \in \mathfrak{T}$ for $\mathbf{r} \in Q$ and act on $\mathfrak{h}^{*}$ as

$$
t_{\mathbf{r}}(\mathbf{v}):=\mathbf{v}+(\mathbf{v} \cdot \boldsymbol{\delta}) \mathbf{r}-\left[(\mathbf{v} \cdot \boldsymbol{\delta}) \frac{1}{2} \mathbf{r}^{2}+\mathbf{r} \cdot \mathbf{v}\right] \boldsymbol{\delta},
$$

where $\mathbf{v} \in \mathfrak{h}^{*}$. Now, it is a well-known result that the affine Weyl group is the semidirect product of the Weyl group of the underlying finite dimensional Lie algebra $\overline{\mathfrak{g}}$ and the affine Weyl translations, i.e.,

$$
\mathfrak{W}(\mathfrak{g})=\mathfrak{W}(\overline{\mathfrak{g}}) \ltimes \mathfrak{T}
$$

To re-examine this result in the light of our approach we need the following family of translations:

$$
\left.{ }^{[\ell}\right]_{\mathbf{r}}(\mathbf{v}) \equiv \mathbf{v}^{\prime}:=\mathbf{v}+\left(\mathbf{v} \cdot \mathbf{k}_{\ell}\right) \mathbf{r}-\left[\left(\mathbf{v} \cdot \mathbf{k}_{\ell}\right) \frac{1}{2} \mathbf{r}^{2}+\mathbf{r} \cdot \mathbf{v}\right] \mathbf{k}_{\ell},
$$

for $\ell \in \mathbb{N}$ and $\mathbf{k} /$ was defined in Eq. (2.3). More specifically, we have the following transformation formulas for a tachyonic level- $\ell$ vector a, a polarization vector $\boldsymbol{\xi}^{i}(\mathbf{a})$, and the affine null vector $\boldsymbol{\delta}$, respectively:

$$
\begin{gathered}
{\left[{ }^{[}\right]_{\mathbf{r}}(\mathbf{a}) \equiv \mathbf{a}^{\prime}=\mathbf{a}+\mathbf{r}-\left(\frac{1}{2} \mathbf{r}^{2}+\mathbf{r} \cdot \mathbf{a}\right) \mathbf{k}_{\ell},} \\
{ }^{\left[{ }^{\prime}\right]} t_{\mathbf{r}}\left(\boldsymbol{\xi}^{i}(\mathbf{a})\right) \equiv \boldsymbol{\xi}^{i}\left(\mathbf{a}^{\prime}\right)=\boldsymbol{\xi}^{i}(\mathbf{a})-\left[\mathbf{r} \cdot \boldsymbol{\xi}^{i}(\mathbf{a})\right] \mathbf{k} /, \\
{\left[{ }^{[}\right]_{\mathbf{r}}(\boldsymbol{\delta}) \equiv \boldsymbol{\delta}^{\prime}=\boldsymbol{\delta} .}
\end{gathered}
$$

The above maps are linear and indeed fulfill the translation property

$$
{ }^{[\ell]} t_{\mathbf{r}^{0}}{ }^{[\ell]} t_{\mathbf{s}}={ }^{[\ell]} t_{\mathbf{r}+\mathbf{s}} \quad \forall \mathbf{r}, \mathbf{s} \in \bar{Q} .
$$

Moreover, they preserve the (Minkowskian!) norm, i.e., $\mathbf{v}^{\prime 2}=\mathbf{v}^{2}$. We will now exploit this fact by re-interpreting them as Lorentz boosts. In this way the affine Weyl group becomes a discrete subgroup of $\operatorname{ISO}(d-2)$, the subgroup of the full Lorentz group $\mathrm{SO}(d-1,1)$ leaving fixed a given lightlike vector. For the level-preserving transformations considered in this section, $\operatorname{ISO}(d-2)$ is therefore nothing but the stability subgroup (in the hyperbolic Weyl group) of the affine null root $\delta$.

To proceed, we rewrite Eq. (4.3) as

$$
v_{\mu}^{\prime}=\left[\bigcap^{\prime} \mathscr{T}_{\mu}{ }^{\nu} v_{\nu}\right.
$$

with

$$
\left.\left[{ }^{[}\right] \mathscr{T}_{\mu}{ }^{\nu} \equiv\left({ }^{[\ell}\right]_{t_{\mathbf{r}}}\right)_{\mu}{ }^{\nu}:=\delta_{\mu}^{\nu}+r_{\mu}\left(\mathbf{k}_{\ell}\right)^{\nu}-\left(\mathbf{k}_{\ell}\right)_{\mu} r^{\nu}-\frac{1}{2} \mathbf{r}^{2}\left(\mathbf{k}_{\ell}\right)_{\mu}\left(\mathbf{k}_{\ell}\right)^{\nu}
$$


It is elementary to show that ${ }^{[\ell]} \mathscr{T}=\exp ^{[\ell]} \omega$ with

$$
{ }^{[\ell]} \omega_{\mu \nu}:=r_{\mu}\left(\mathbf{k}_{\ell}\right)_{\nu}-r_{\nu}\left(\mathbf{k}_{\ell}\right)_{\mu} .
$$

We have a unitary representation $[\leftrightharpoons \hat{\mathscr{T}}$ of this Lorentz boost on the Fock space by means of the Lorentz generators (3.16), viz.

$$
\left[\ell \hat{\mathscr{T}}:=\exp \left(\frac{i}{2}\left[{ }^{[/]} \omega_{\mu \nu} M^{\mu \nu}\right)\right.\right.
$$

one finds that

$$
\left[\ell \hat{\mathscr{T}}(\boldsymbol{\lambda} \cdot \mathbf{q})\left({ }^{[\ell]} \hat{\mathscr{T}}\right)^{-1}={ }^{[\ell]} t_{\mathbf{r}}(\boldsymbol{\lambda}) \cdot \mathbf{q}, \quad[\ell] \hat{\mathscr{T}}\left(\boldsymbol{\lambda} \cdot \boldsymbol{\alpha}_{m}\right)\left({ }^{[\ell]} \hat{\mathscr{T}}\right)^{-1}={ }^{[\ell]} t_{\mathbf{r}}(\boldsymbol{\lambda}) \cdot \boldsymbol{\alpha}_{m} \quad \forall m \in \mathbb{Z} .\right.
$$

For instance, on the tachyon state $|\mathbf{a}\rangle$, we get

$$
\left[\ell \hat{\mathscr{T}}|\mathbf{a}\rangle=\left|\mathbf{a}^{\prime}\right\rangle .\right.
$$

Since, as we already noted, the transverse DDF oscillators remain unchanged for $m \neq 0$, we therefore have, on $\mathscr{P}(\boldsymbol{\Lambda})$,

$$
\begin{gathered}
{[\ell] \hat{\mathscr{T}} A_{m}^{i}(\mathbf{a})([\ell] \hat{\mathscr{T}})^{-1}=A_{m}^{i}\left(\mathbf{a}^{\prime}\right)=A_{m}^{i}(\mathbf{a}),} \\
{\left[\ell \hat { \mathscr { T } } A _ { 0 } ^ { i } ( \mathbf { a } ) \left([\ell \hat{\mathscr{T}})^{-1}=A_{0}^{i}\left(\mathbf{a}^{\prime}\right)=A_{0}^{i}(\mathbf{a})-\mathbf{r} \cdot \boldsymbol{\xi}^{i}(\mathbf{a}) .\right.\right.}
\end{gathered}
$$

Although the replacement of the momentum shift by the Weyl translation (4.3) and the re-interpretation of this translation as a Lorentz boost was forced on us by the replacement of ordinary string oscillators by DDF operators, it is now clear that this interpretation is the natural one. This is also evident from the following diagram, which displays the nested sequence of Weyl groups of the finite, affine and hyperbolic Kac-Moody algebras $\overline{\mathfrak{g}} \subset \mathfrak{g} \subset \hat{\mathfrak{g}}$ as discrete subgroups of the corresponding continuous groups

$$
\begin{array}{ccccc}
\mathfrak{W}(\overline{\mathfrak{g}}) & \subset & \mathfrak{W}(\mathfrak{g}) & \subset & \mathfrak{W}(\hat{\mathfrak{g}}) \\
\cap & & \cap & & \cap \\
\operatorname{SO}(d-2) & \subset & \operatorname{ISO}(d-2) & \subset & \operatorname{SO}(d-1,1)
\end{array}
$$

We can thus think of the affine Weyl group as a dimensional null reduction of the full hyperbolic Weyl group, similar in spirit to the Kaluza-Klein reduction of Einstein's theory with a nullKilling vector which was recently studied in Ref. 13, where the group $\operatorname{ISO}(d-2)$ made its appearance as the residual tangent space symmetry.

\section{LONGITUDINAL DDF OPERATORS AND LEVEL-CHANGING TRANSFORMATIONS}

We finally turn to longitudinal DDF operators. In the representation theory of affine algebras these have played no role so far, because one usually considers only one representation at a time. By contrast, the longitudinal DDF operators do change the level, and therefore interpolate between different, and inequivalent affine representations. This fact is immediately evident if one allows the vector $\mathbf{r}$ in the exponent of Eq. (3.20) to have level $\ell \neq 0$, in which case the field $\mathscr{C}^{\mu}$ inevitably acquires a longitudinal component. Conversely, a nonvanishing longitudinal component in this expression implies that $\mathbf{r}$ cannot only have components in the affine root lattice, but must have $\ell \neq 0$. While the necessity of studying several representations simultaneously does not arise in the theory of affine representations as such, the problem must be faced when one considers hyperbolic Kac-Moody algebras which contain infinitely many affine representations of arbitrary 
level. Furthermore, as shown in Ref. 8, longitudinal states do appear in these algebras. We claim that the formalism developed in this paper furnishes the requisite tools for further investigations in this direction, because it allows us to embed the different representations into a single Fock space of physical states. In this section we present some preliminary results concerning the longitudinal DDF operators, which we expect to become relevant in future developments. In particular, we will also consider the level-changing generalizations of the Lorentz boosts (4.9), whose unitary implementation yields operators interpolating between DDF operators of different level.

Because there is an infinity of tachyonic states $|\mathbf{a}\rangle$, the longitudinal DDF operators $A_{m}^{-}(\mathbf{a})$ introduced in Eq. (2.10) constitute an infinity of Virasoro algebras, but with uniform central charge $c=26-d$, see Eq. (2.17). By construction, all of these commute with the Virasoro generators $L_{m}$, and are therefore physical. Moreover, they also commute with the Sugawara generators $(3.22)$

$$
\left[\mathscr{L}_{m}^{[\ell]}, A_{n}^{-}(\mathbf{a})\right]=0
$$

for all tachyonic a associated with a weight $\boldsymbol{\lambda} \in \Omega(\boldsymbol{\Lambda})$. Although their polarization is along a, a short calculation shows that they are still invariant under the Lorentz boost (4.8), in accordance with Eqs. (4.14) and (4.15); namely, we have

$$
\begin{gathered}
{\left[\ell \hat{\mathscr{T}} A_{m}^{-}(\mathbf{a})([\ell] \hat{\mathscr{T}})^{-1}=A_{m}^{-}\left(\mathbf{a}^{\prime}\right)=A_{m}^{-}(\mathbf{a}),\right.} \\
{[\ell] \hat{\mathscr{T}} A_{0}^{-}(\mathbf{a})\left([\ell \hat{\mathscr{T}})^{-1}=A_{0}^{-}\left(\mathbf{a}^{\prime}\right)=A_{0}^{-}(\mathbf{a})+\mathbf{r} \cdot \mathbf{a} .\right.}
\end{gathered}
$$

Their commutation relations with the step operators (3.20) (for $r \in \bar{\Delta}$ ) are given by

$$
\hat{E}_{m}^{\mathbf{r}} A_{n}^{-}(\mathbf{a})=A_{n}^{-}\left(\mathbf{a}^{\prime}\right) \hat{E}_{m}^{\mathbf{r}},
$$

where $\mathbf{a}^{\prime}$ is the Weyl-boosted tachyon momentum defined in Eq. (4.4). In deriving this result, the $A^{i} A^{i}$ term in Eq. (2.10) is essential. The longitudinal DDF operators can thus be regarded as intertwining operators between different (but isomorphic) representations. The above relation also permits us to extend the proof of Theorem 1 to states containing longitudinal excitations by simply moving all step operators to the right of the longitudinal DDF operators.

So far, we have only considered the action of integrated tachyon vertex operators associated with affine roots $\mathbf{r}+m \boldsymbol{\delta} \in \Delta$. From the point of view of string theory it is natural to incorporate "step operators" associated with arbitrary tachyonic affine dominant integral weights $\boldsymbol{\Lambda}^{\prime}$. So let us define

$$
E^{\Lambda^{\prime}}:=\oint \frac{d z}{2 \pi i}: \exp \left[i \Lambda^{\prime} \cdot \mathbf{X}(z)\right]: c_{\Lambda^{\prime}}
$$

for $\Lambda^{\prime} \in P_{+}$satisfying $\Lambda^{\prime 2}=2$. Since $\Lambda^{\prime}$ has nonvanishing level in general, only the special case of level zero $\left(\boldsymbol{\Lambda}^{\prime}=\mathbf{r}+m \boldsymbol{\delta}\right.$ for $\left.\mathbf{r} \in \bar{\Delta}\right)$ leads to the step operators $E_{m}^{\mathbf{r}}$. By construction, the generalized step operators are physical,

$$
E^{\boldsymbol{\Lambda}^{\prime}}: \mathscr{P}^{(\boldsymbol{\lambda})} \rightarrow \mathscr{P}^{\left(\boldsymbol{\lambda}+\boldsymbol{\Lambda}^{\prime}\right)} ;
$$

but the crucial observation is that they change the level, i.e., they map from a highest weight module $L(\boldsymbol{\Lambda})$ to the module $L\left(\boldsymbol{\Lambda}+\boldsymbol{\Lambda}^{\prime}\right)$. Again, one might wonder whether these operators can be rewritten in a manifestly physical form, and it is at this point that the longitudinal DDF operators will enter the stage. In the remainder, we will therefore generalize the affine Weyl translations to level-changing translations which will be necessary for rewriting the generalized step operators in terms of the DDF operators. 
For any $\boldsymbol{\lambda}_{1}$ in the weight system $\Omega\left(\Lambda_{1}\right)$ of a dominant weight $\Lambda_{1}$ with positive level $\ell_{1}$, we define

$$
{ }^{[}{ }^{[} t_{\boldsymbol{\lambda}_{1}}(\mathbf{v}):=\mathbf{v}+\left(\mathbf{v} \cdot \mathbf{k}_{\ell}\right) \boldsymbol{\lambda}_{1}-\left[\left(\mathbf{v} \cdot \mathbf{k}_{\ell}\right) \frac{1}{2} \boldsymbol{\lambda}_{1}^{2}+\mathbf{v} \cdot \boldsymbol{\lambda}_{1}\right] \mathbf{k}_{\ell+\ell_{1}} .
$$

Note that the affine null root is no longer invariant but is rescaled according to

$$
{ }^{[}{ }^{\left[\boldsymbol{\lambda}_{1}\right.}{ }_{\mathbf{\lambda}_{\ell}}\left(\mathbf{k}_{\ell}\right) \mathbf{k}_{\ell+\ell_{1}} .
$$

The above maps are linear and again fulfill the translation property

$$
{ }^{[\ell+\ell}{ }^{1}{ }_{\lambda_{2}}{ }^{\circ}{ }^{[\ell]} t_{\lambda_{1}}={ }^{[\ell]} t_{\lambda_{1}+\lambda_{2}}
$$

Moreover, they preserve the norm, which allows us to rewrite them as Lorentz boosts. To see this, we rewrite Eq. (5.5) as

$$
\left[{ }^{[\ell]} t_{\mathbf{\lambda}_{1}}(\mathbf{v})\right]_{\mu}{ }^{\nu} v_{\nu}={ }^{[\ell]} \mathscr{T}_{\mu}{ }^{\nu} v_{\nu}
$$

with

$$
{ }^{[\ell]} \mathscr{T}_{\mu}^{\nu} \equiv\left({ }^{[\ell]} t_{\boldsymbol{\lambda}_{1}}\right)_{\mu}{ }^{\nu}:=\delta_{\mu}^{\nu}+\left(\boldsymbol{\lambda}_{1}\right)_{\mu}\left(\mathbf{k}_{\ell}\right)^{\nu}-\left(\mathbf{k}_{\ell+\ell_{1}}\right)_{\mu}\left(\boldsymbol{\lambda}_{1}\right)^{\nu}-\frac{1}{2} \boldsymbol{\lambda}_{1}^{2}\left(\mathbf{k}_{\ell+\ell_{1}}\right)_{\mu}\left(\mathbf{k}_{\ell}\right)^{\nu} .
$$

A careful calculation shows that ${ }^{[/]} \mathscr{T}=\exp ^{[\ell]} \omega$ with

$$
{ }^{\left[{ }^{\prime}\right.} \omega_{\mu \nu}:=\ln \left(1+\frac{\ell}{\ell}\right)\left[\left(\boldsymbol{\lambda}_{1}\right)_{\mu}\left(\mathbf{k}_{\ell_{1}}\right)_{\nu}-\left(\mathbf{k}_{\ell_{1}}\right)_{\mu}\left(\boldsymbol{\lambda}_{1}\right)_{\nu}\right] .
$$

The last two equations generalize the expressions in Eqs. (4.9) and (4.10), respectively, which can be reobtained by putting $\ell_{1}=0$. The unitary representation $[\ell] \hat{\mathscr{T}}$ of this level-changing Lorentz boost on the Fock space is still given by formula (4.11). By Eq. (5.6), conjugation with the operator ${ }^{[\ell]} \hat{\mathscr{T}}$ transmutes level- $\ell$ DDF operators into level- $\left(\ell+\ell_{1}\right)$ DDF operators.

A natural ansatz for a longitudinal coordinate field analogous to Eq. (3.15) is

$$
\mathscr{X}_{\boldsymbol{\Lambda}, \boldsymbol{\Lambda}_{1}}^{-}(z):=\ln \left(1+\frac{\ell}{\ell}\right)\left(\boldsymbol{\Lambda}_{1}\right)_{\mu}\left(\mathbf{k}_{\ell_{1}}\right)_{\nu} M^{\mu \nu}-i\left(\boldsymbol{\Lambda}_{1} \cdot \mathbf{p}\right) \ln z+i \sum_{m \neq 0} \frac{1}{m} A_{m}^{-}\left(\boldsymbol{\Lambda}+\boldsymbol{\Lambda}_{1}\right) z^{-m},
$$

with associated longitudinal momentum field

$$
\mathscr{P}_{\mathbf{\Lambda}, \mathbf{\Lambda}_{1}}^{-}(z):=i \frac{d}{d z} \mathscr{C}_{\mathbf{\Lambda}, \boldsymbol{\Lambda}_{1}}^{-}(z)=\sum_{m \in Z} A_{m}^{-}\left(\boldsymbol{\Lambda}+\boldsymbol{\Lambda}_{1}\right) z^{-m-1} .
$$

This means, however, that one will have to face up to the problem of dealing with exponentials of such operators. This is not quite the same as exponentiating the Virasoro algebra because our operators are always well defined on finite occupation number states, for which the contour integral picks up only finitely many contributions, but the technical problems of manipulating such expressions (e.g., computing operator products analogous to the ones used in the proof of Theorem 1) still seem daunting. The relevant calculations would be analogous to the computation of string scattering amplitudes in the light cone gauge, with the longitudinal operators and bilinears of transversal oscillators in the exponent-something that apparently has never been done in the literature. 


\section{ACKNOWLEDGMENTS}

We would like to thank J. Fuchs for careful reading of the manuscript. R.W.G. was supported by the Deutsche Forschungsgemeinschaft under Contract No. DFG Ge 963/1-1.

${ }^{1}$ I. B. Frenkel and V. G. Kac, "Basic representations of affine Lie algebras and dual models,”' Invent. Math. 62, 23-66 (1980).

${ }^{2}$ G. Segal, "Unitary representations of some infinite dimensional groups," Commun. Math. Phys. 80, 301-342 (1981).

${ }^{3}$ I. B. Frenkel, "Representations of Kac-Moody algebras and dual resonance models," in Applications of Group Theory in Theoretical Physics (American Mathematical Society, Providence, RI, 1985), pp. 325-353.

${ }^{4}$ P. Goddard and D. Olive, "Algebras, lattices and strings," in Vertex Operators in Mathematics and PhysicsProceedings of a Conference, 10-17 November 1983, edited by J. Lepowsky, S. Mandelstam, and I. M. Singer (Springer, New York, 1985), pp. 51-96.

${ }^{5}$ R. W. Gebert, K. Koepsell, and H. Nicolai, “The Sugawara generators at arbitrary level,” Commun. Math. Phys. 184 119-141 (1997).

${ }^{6}$ V. G. Kac, Infinite Dimensional Lie Algebras, 3rd ed. (Cambridge University Press, Cambridge, 1990).

${ }^{7}$ R. V. Moody and A. Pianzola, Lie Algebras With Triangular Decomposition (Wiley, New York, 1995).

${ }^{8}$ R. W. Gebert and H. Nicolai, "On $E_{10}$ and the DDF construction," Commun. Math. Phys. 172, 571-622 (1995).

${ }^{9}$ E. Del Giudice, P. Di Vecchia, and S. Fubini, "General properties of the dual resonance model," Ann. Phys. 70, 378-398 (1972).

${ }^{10}$ R. C. Brower, "Spectrum-generating algebra and no-ghost theorem for the dual model," Phys. Rev. D 6, 1655-1662 (1972).

${ }^{11}$ V. Marotta, "Relation between covariant and parafermionic construction of affine Kac-Moody algebras," J. Phys. A 26, 3481-3487 (1993).

${ }^{12}$ P. Goddard and D. Olive, "Kac-Moody and Virasoro algebras in relation to quantum physics," Int. J. Mod. Phys. A 1, 303-414 (1986).

${ }^{13}$ B. Julia and H. Nicolai, "Null-Killing vector dimensional reduction and Galilean geometrodynamics," Nucl. Phys. B 439, 291-323 (1995). 